\title{
The Impact of Levothyroxine in Women with Positive Thyroid Antibodies on Pregnancy Outcomes: A Systematic Review and Meta-Analysis of Randomized Controlled Trials
}

\author{
Lorraine Lau ${ }^{1}$, Jamie Benham ${ }^{1}$, Patricia Lemieux ${ }^{1}$, Jennifer Yamamoto ${ }^{1}$, and Lois \\ Donovan $^{2}$ \\ ${ }^{1}$ University of Calgary Cumming School of Medicine \\ ${ }^{2}$ University of Calgary
}

July 23, 2020

\begin{abstract}
Background: Thyroid autoimmunity in pregnant women has been associated with negative outcomes. Objective: To evaluate the effect of levothyroxine therapy on pregnancy outcomes compared with placebo or no treatment in women without overt hypothyroidism who are TPOAb and/or TgAb-positive. Search Strategy: Ovid MEDLINE, EMBASE, CINAHL, Cochrane Database of Systematic Reviews, and Cochrane Central Register of Controlled Trials were searched from 1980 to April 17, 2019. Selection Criteria: Pre-specified criteria for inclusion were: randomized trials of levothyroxine versus control (placebo or no treatment) among women with positive TPOAb or TgAb who were pregnant or considering conception. Data Collection and Analysis: Pre-specified data elements were extracted and where appropriate, meta-analyses were conducted. Main outcomes include pregnancy achieved, miscarriage, preterm delivery and live birth. Main Results: From 2,812 citations, 79 citations were identified for full text review. Of these, six trials (total of 2,263 women) were included for qualitative and quantitative analyses. Risk of bias was deemed low for only one trial. There was no significant difference in the relative risk (RR) of pregnancy achieved (RR 1.03; 95\% CI 0.93, 1.13), miscarriage (RR 0.93; 95\% CI 0.76, 1.14), preterm delivery (RR 0.66; 95\% CI $0.39,1.10$ ), or live births (RR 1.01; 95\% CI 0.89, 1.16) in thyroid autoimmune women treated with levothyroxine compared to controls. Conclusion: Among pregnant women or women planning conception, with thyroid autoimmunity, there is a lack of evidence of benefit for levothyroxine use. Recommendations to use levothyroxine in this setting need to be reconsidered.
\end{abstract}

\section{INTRODUCTION:}

Thyroid autoimmunity, defined by the presence of thyroid peroxidase antibodies (TPOAb) and/or thyroglobulin antibodies $(\mathrm{TgAb})$, is relatively common in pregnant women. The prevalence of TPOAb positivity in the first trimester ranges from 5 to $15 \%$ in unselected pregnant women $(1,2)$. The prevalence of thyroid autoimmunity is even higher in women with a history of recurrent pregnancy loss, reported as 23 to $31 \%$ (3, 4).

The association between thyroid autoimmunity and poor pregnancy outcomes, specifically miscarriage and preterm birth, has been well described $(5,6)$. However, the mechanism linking thyroid autoimmunity with increased risk of miscarriage and preterm birth in women without overt hypothyroidism is unclear. Two competing mechanistic theories propose that 1) thyroid autoantibodies are a marker of a hostile immune environment, or 2) thyroid autoantibodies act as a surrogate biomarker of impaired thyroid hormone reserves, despite normal thyroid function tests(7). If the latter theory is true, one could postulate that supplementation with levothyroxine may improve thyroid hormone levels and prevent negative pregnancy outcomes.

Whether or not levothyroxine therapy, either prior to or during pregnancy, improves pregnancy outcomes in 
women with thyroid autoimmunity is controversial. The 2017 American Thyroid Association (ATA) pregnancy guidelines strongly recommends levothyroxine for women with thyroid autoimmunity and a thyroid stimulated hormone (TSH) above the pregnancy-specific reference range. These guidelines recommend a consideration of levothyroxine in thyroid autoimmune women with TSH above $2.5 \mathrm{mIU} / \mathrm{L}$ and among euthyroid women with positive TPOAb and a history of pregnancy loss(8). The evidence evaluating levothyroxine treatment in pregnant women with thyroid autoantibodies is conflicting. Additionally, the limitations of observational studies must be considered. Observational studies identify associations, but do not provide the highest level of evidence for determining causation or therapeutic efficacy. Randomized controlled trials are considered the gold standard to evaluate therapeutic efficacy $(9)$. Therefore, we performed a systematic review and meta-analysis to evaluate all randomized controlled trials of levothyroxine therapy in women with thyroid autoimmunity who were pregnant or planning pregnancy.

\section{METHODS}

\section{Protocol and Registration}

A systematic review and meta-analysis was performed as outlined in the registered protocol (PROSPERO CRD42019130459), and is reported in accordance with the Preferred Reporting Items for Reviews and MetaAnalyses (PRISMA).

\section{Data Sources and Search Strategy}

The following databases were searched in duplicate on April 17, 2019 from 1980: Ovid MEDLINE, EMBASE, CINAHL, Cochrane Database of Systematic Reviews, and Cochrane Central Register of Controlled Trials. Clinicaltrials.gov was searched for active trials. The search strategy was developed in consultation with a medical librarian and used a combination of keywords related to hypothyroidism or thyroid autoimmunity and pregnancy (Supplemental S1). The search was conducted using an adapted version of the Cochrane search filter for randomized controlled trials where possible and was limited to human studies(10).

\section{Study Selection}

Studies that met all the following criteria were included in this review: (1) pregnant women or women contemplating pregnancy (including patients seeking assisted reproductive technologies), with positive TPOAb or TgAb, euthyroid or with subclinical hypothyroidism (by any prespecified study definition); (2) randomized to levothyroxine versus control (placebo or no treatment); (3) reported pregnancy outcomes; (4) reported in either English or French; (5) published from 1980 to April 17, 2019. The publication year 1980 was specifically chosen to coincide with the availability of more sensitive TSH assays(11, 12).

Study selection was completed in two separate stages by two independent reviewers (PL and LED or LWL and JLB). First, duplicates were removed, and titles and abstracts were screened for eligibility (by PL and LED). Second, full citations deemed eligible at the title and abstract stage were retrieved and reviewed for eligibility in a full-text format (by LWL and JLB). Inclusion criteria was applied to select eligible articles and reasons for exclusion were recorded. Agreement was recorded at each stage and reported as a kappa statistic(13). All disagreements between reviewers were solved by consensus, and, if needed, by discussion with a third independent reviewer (LED).

\section{Data Collection Process}

Relevant study information was extracted by two independent reviewers (LWL and JLB) using standardized data extraction forms in Microsoft Excel (Version 16.16.18, Microsoft Corporation, Redmon, WA, USA). Extracted data elements included: study design, country, participant inclusion and exclusion criteria, TPOAb and TgAb results, definition of TPOAb and TgAb positivity, TPOAb and TgAb assays used, thyroid stimulating hormone (TSH) and thyroxine (T4) assays used and normal ranges, baseline serum concentrations of TSH and T4, iodine status of the country, levothyroxine dosing, achieved TSH and T4 after treatment, gestational timing of levothyroxine initiation, duration of treatment, details of the intervention, quality of the methods, inclusion of patients with subclinical hypothyroidism and definition of subclinical hypothyroidism, 
patient baseline characteristics, number of patients included in each group, pregnancy outcomes and their definitions.

\section{Quality Assessment}

Cochrane Risk of Bias Tool (Modified) for Quality Assessment of Randomized Controlled Trials(14) was used to assess study quality and evaluate the risk of bias by two independent reviewers (LL and JB). This tool assesses seven methodologic domains and these components enable classification of randomized trials as "Low Risk of Bias", "High Risk of Bias" or "Unclear Risk of Bias".

\section{Study Outcomes}

The following pregnancy outcomes were prespecified and included: pregnancy by trial definition, miscarriage, preterm delivery, live-birth, birth weight, large for gestational age, small for gestational age, gestational age at delivery, multiple pregnancy, stillbirth, neonatal intensive care unit admission, preeclampsia, and gestational diabetes.

\section{Data Synthesis and Analysis}

Where appropriate, meta-analyses were conducted to evaluate the relative risk of pre-specified pregnancy outcomes including pregnancy achieved, miscarriage, preterm delivery and live-birth in participants randomized to levothyroxine therapy compared with placebo or no treatment. Random-effect models were used. Relative risk (RR) and 95\% confidence intervals (95\% CI) were calculated. Statistical heterogeneity was quantified using $\mathrm{I}^{2}$ statistics, where $\mathrm{I}^{2}>50 \%$ represents moderate and $\mathrm{I}^{2}>75 \%$ represents substantial heterogeneity across studies. The following prespecified sensitivity analyses were conducted to explore heterogeneity: 1) TSH levels at baseline, 2) time of levothyroxine initiation (preconception or during pregnancy), 3) use and type of assisted reproduction technologies, 4) maternal characteristics, and 5) study quality. Publication bias was assessed using graphical aids. Statistical analysis was performed using STATA version 14 (StataCorp, College Station, TX, USA) and Review Manager 5.3 (Version 5.3.5, The Cochrane Collaboration, Copenhagen, Denmark).

\section{RESULTS}

The search results are summarized in Figure 1. Among the 2,812 citations reviewed for title and abstract, 79 citations were identified for full text review. Six randomized controlled trials (total of 2,263 women) met all eligibility criteria and were included in the qualitative and quantitative analyses(15-20). There was a substantial level of agreement for both the title and abstract review $(\varkappa=0.64)$ and for the full text review $(x=0.70)$.

\section{Trial Characteristics}

Trial characteristics are summarized in Table 1. Three trials initiated levothyroxine therapy prior to concep$\operatorname{tion}(15,18,20)$, whereas three trials initiated therapy in the first trimester $(16,17,19)$. Two trials compared levothyroxine to placebo $(15,17)$, and the other four trials compared with no treatment(16, 18-20). Reproductive technologies were used in three trials $(15,18,20)$, with one trial not specifying details with this regard. All trials included women with positive TPOAb with only one trial including TPO or Tg antibodies(20). The TSH normal reference ranges and definition of TPO positivity were prespecified in each trial and are summarized in Table 1.

\section{Patient Characteristics}

Baseline participant characteristics are summarized in Table 1 and Supplemental S2. Mean maternal age ranged from 26 years(16) to 32 years(15). Mean reported body mass index ranged from $22.7 \mathrm{~kg} / \mathrm{m}^{2}(20)$ to $26.5 \mathrm{~kg} / \mathrm{m}^{2}{ }^{(15)}$. Baseline TSH values varied according to assay specific reference ranges. Baseline free T4 values were comparable in the three trials that reported this information(15, 18, 19) (Supplemental S2).

\section{Risk of Bias Assessment}


Risk of bias assessment is presented in Figure 2. Only one trial(15) was considered "low risk" of bias in all seven domains. Four trials(15, 17-19) were assessed at "low risk" for selection bias. Two trials(16, 20) were "unclear risk of bias" in allocation concealment. In four trials comparing levothyroxine to no treatment(16, $17,19,20)$, the assessors were not blinded to patient randomization and thus these trials were considered to be "high risk" for performance bias. Published trial protocols were located for only two trials $(15,20)$. Thus, only these two trials were assessed as "low risk" for reporting bias.

\section{Pregnancy Outcomes}

There were sufficient data for meta-analyses of four pregnancy outcomes, as summarized in Figure 3. These outcomes include pregnancy achieved, miscarriage, preterm delivery and live births.

Pregnancy achieved was reported in three trials $(15,18,20)(n=1,626)$ that initiated levothyroxine preconception. There was no significant difference in pregnancy achieved in thyroid autoimmune women treated levothyroxine compared with control (RR 1.03 [95\% CI 0.93, 1.13]; $\mathrm{I}^{2}=0 \%$ )

Miscarriage was reported in all six trials $(n=1,427)$ and was defined as pregnancy loss at less than 20 weeks(16), less than 24 weeks(15), less than 28 wks (early miscarriage defined as first 12 weeks, late miscarriage defined as between 13-28 weeks)(20), or not clearly defined(17-19). There was no significant difference in the relative risk (RR) of miscarriage in TPOAb-positive pregnant women treated with levothyroxine compared with control (RR 0.93 [95\% CI 0.76, 1.14]; $\mathrm{I}^{2}=0 \%$ ). Inclusion of trials $(15,18,20)$ that initiated levothyroxine only preconception revealed no significant difference compared with control (RR 0.91 [95\% CI $\left.0.72,1.15] ; \mathrm{I}^{2}=0 \%\right)$.

Preterm delivery was reported in five trials $(\mathrm{n}=1,354)(15-17,19,20)$. Preterm delivery was defined as live birth prior to 34 weeks $(15)$ or 37 weeks $(16,17,19,20)$. There was no significant difference in the relative risk of preterm delivery in thyroid autoimmune pregnant women treated with levothyroxine compared with control (RR 0.66 [95\% CI 0.39, 1.10]; I ${ }^{2}=53 \%$ ).

Live births were reported in two trials $(\mathrm{n}=1,540)(15,20)$. Live birth was defined as delivery of a living fetus beyond 27 weeks(20) or beyond 34 weeks(15). There was no significant difference in live births in thyroid autoimmune women treated with levothyroxine compared with control (RR 1.01 [95\% CI 0.89, 1.16]; $\mathrm{I}^{2}=$ $0 \%)$.

Only one trial(17) reported on pre-eclampsia and found no difference in this outcome among thyroid autoimmune women treated with levothyroxine compared with control. None of the studies reported the outcome of gestational diabetes.

\section{Neonatal Outcomes:}

Neonatal outcomes were only reported in two trials $(\mathrm{n}=1,083)(15,16)$. Meta-analysis for gestational age at time of delivery (measured in days) and birth weight (measured in grams) are summarized in Supplemental S3. The weighted mean difference for gestational age at time of delivery in women treated with levothyroxine compared with placebo or no treatment was 2.35 days $\left(95 \%\right.$ CI $\left.-4.51,9.20 ; \mathrm{I}^{2}=90 \%\right)$. Weighted mean difference for birth weight in women treated with levothyroxine compared with placebo or no treatment was -24.05 g (95\% CI -96.91, 48.80; $\left.\mathrm{I}^{2}=0 \%\right)$.

Only one trial(16) reported neonatal intensive care unit admission. They found fewer admissions among thyroid autoimmune women treated with levothyroxine (2 of 56 compared with control 12 of $58, \mathrm{p}<0.05$ ). No studies reported large for gestational age, small for gestational age or multiple pregnancy.

Sensitivity Analyses A sensitivity analysis was performed to explore the heterogeneity of our meta-analysis for preterm delivery. Results are summarized in Supplemental S4.

TSH levels at baseline were subdivided into TSH [?] $2.5 \mathrm{mIU} / \mathrm{mL}(15,17,19)$ and TSH $>2.5 \mathrm{mIU} / \mathrm{mL}(16$, 20). The relative risk of preterm delivery in pregnant women with baseline TSH [?] $2.5 \mathrm{mIU} / \mathrm{mL}$ was 0.64 
$\left(95 \%\right.$ CI $\left.0.36,1.14 ; \mathrm{I}^{2}=32 \%\right)$ compared with a relative risk of $0.62\left(95 \%\right.$ CI $\left.0.17,2.33 ; \mathrm{I}^{2}=80 \%\right)$ in pregnant women with baseline TSH $>2.5 \mathrm{mIU} / \mathrm{mL}$.

Timing of levothyroxine initiation was stratified into preconception $(15,20)$ and in pregnancy $(16,17,19)$. The relative risk of preterm delivery in women initiated on levothyroxine preconception was 1.10 (95\% CI $\left.0.69,1.75 ; \mathrm{I}^{2}=0 \%\right)$ compared a relative risk of $0.46\left(95 \%\right.$ CI $\left.0.28,0.75 ; \mathrm{I}^{2}=2 \%\right)$ in women initiated on levothyroxine in the first trimester.

The relative risk of preterm delivery in women using assisted reproductive technologies (ART) $(15,20)$ was $1.10\left(95 \%\right.$ CI $\left.0.69,1.75, \mathrm{I}^{2}=0 \%\right)$ compared with a relative risk of $0.51\left(95 \%\right.$ CI $\left.0.26,1.02 ; \mathrm{I}^{2}=27 \%\right)$ in women that did not use $\operatorname{ART}(17,19)$.

Sensitivity analyses was also performed to explore the impact of fixed $(15,18)$ and adjusted levothyroxine(16, $17,19,20)$ doses on miscarriage rates (Supplemental S5). The relative risk of fixed levothyroxine dose was $0.89\left(95 \%\right.$ CI $\left.0.66,1.20 ; \mathrm{I}^{2}=12 \%\right)$ compared with adjusted levothyroxine dose of 0.95 (95\% CI $0.68,1.33 ; \mathrm{I}^{2}$ $=0 \%)$.

Study quality was defined as high if there was low risk of bias across all seven domains of the Cochrane Collaboration's tool. We were unable to perform a sensitivity analysis exploring the impact of risk of bias on preterm delivery, as only one trial met the definition for high quality per the Cochrane Collaboration tool(15). A funnel plot for preterm delivery is shown in Supplemental S6. On visual inspection, there was asymmetry indicating potential publication bias.

\section{DISCUSSION}

\section{Main Findings}

This systematic review and meta-analysis of randomized controlled trials of levothyroxine therapy in women with thyroid autoimmunity without overt thyroid dysfunction demonstrates no evidence of benefit of levothyroxine therapy across prespecified pregnancy outcomes. Specifically, treatment with levothyroxine did not improve pregnancies achieved, miscarriage preterm delivery or live-birth across a total of 2,263 women, inclusive of those with a history of pregnancy loss and use of reproductive technologies. Furthermore, the availability of larger and better-quality trials since the publications of the American Thyroid Association 2017 pregnancy recommendations signals the need to update these guidelines as they pertain to the treatment of thyroid autoimmune women.

\section{Interpretation}

Small randomized clinical trials initially demonstrated that levothyroxine therapy reduced miscarriage and/or pre-term delivery in TPOAb-positive women compared with placebo or no treatment intervention(16-18). However, the unclear definition of miscarriage and pre-term delivery among these early trials means that selective reporting of outcomes could have biased their reported findings. Subsequent large, multi-center randomized controlled trials with clear prespecified outcome definitions have shown no impact of levothyroxine therapy on maternal or fetal outcomes compared with no levothyroxine treatment in pregnant women with subclinical hypothyroidism, isolated hypothyroxinemia or positive $\operatorname{TPOAb}(15,20-22)$. These intervention trials involved pregnant women or women seeking pregnancy, including individuals with a history of pregnancy loss.

A systematic review and meta-analysis of a large study population $(\mathrm{n}=47,045)$ of individual participant and population data demonstrated increased risk of preterm birth in TPOAb-positive women ${ }^{6}$. In light of these findings, Cappola and Casey(23) highlighted the need to address whether levothyroxine treatment decreases the risk of preterm birth among women with TPOAb. Three other systematic reviews and meta-analyses examined the effect of levothyroxine supplementation in pregnant women with thyroid autoimmunity $(24-26)$. Unlike our review which only included randomized controlled trials, two of the other reviews ${ }^{24,25}$ included observational studies. The findings of our meta-analysis were consistent with the review by Sun et al .(24), which showed no benefit of levothyroxine supplementation on their pregnancy outcomes. In contrast, Rao 
et al. $(25,26)$ demonstrated a beneficial effect of levothyroxine treatment on reducing pregnancy loss and preterm birth among pregnant women with subclinical hypothyroidism and/or thyroid autoimmunity. Several notable distinctions likely account for these discrepant results. First, the Dhillon-Smith et al. (15) publication was not included is these reviews. Furthermore, both reviews by Rao et al. $(25,26)$ included a trial that strongly influenced their results but did not report outcome data in sufficient detail to be included in our systematic review(27). Our attempts to clarify the outcome data of this trial(27) with the authors were left unresponded.

Most miscarriages occur early in pregnancy, prior to the first obstetrics appointment, thus initiation of levothyroxine prior to conception best addresses its impact on miscarriage rates. The pathophysiology underlying the association between thyroid autoimmunity in euthyroid pregnant women and miscarriage is still not completely understood. One hypothesis postulates that thyroid autoimmunity reflects inadequate thyroid reserves to accommodate for increased physiologic demands of pregnancy(28). It has been postulated that delayed initiation of levothyroxine in pregnancy may account for its failure to reduce miscarriage. Our meta-analysis found that initiation of levothyroxine preconception or in the first trimester did not impact miscarriage outcomes. This suggests that inadequate thyroid reserve in women with thyroid autoimmunity does not account for the increased risk for miscarriage. A highly anticipated trial T4-LIFE that has not yet been published may further address the role of levothyroxine administration in women with TPOAb and recurrent miscarriage $(29)$.

Sensitivity analyses based on timing of levothyroxine initiation demonstrated a reduction in preterm delivery when levothyroxine was initiated in the first trimester rather than preconception. However, this finding is likely attributed to the quality of the included studies since many of the trials that initiated levothyroxine in the first trimester were at high risk of bias and the funnel plot suggests publication bias. Furthermore, it appears biologically implausible that levothyroxine would be beneficial for thyroid autoimmune women when initiated during pregnancy but not when initiated preconception.

\section{Strengths and Limitations}

A limitation of this study is that only one of the six included trials met all criteria for low risk of bias. Meta-analyses of live births and neonatal outcomes such as gestational age at delivery and birth weight were limited as only two trials reported these outcomes $(15,16)$. Furthermore, the sensitivity analysis is limited by the small number of studies included.

This study has many notable strengths. First, this study provides an updated summary of randomized controlled trials not included in previous systematic reviews and meta-analyses or available at the time of the American Thyroid Association 2017 pregnancy guideline recommendations development. The exclusion of observational studies minimizes bias and inclusion of only randomized controlled trials provides strong evidence to guide clinical decisions. Our systematic review and meta-analysis benefits from the use of rigorous standardized tools such as Cochrane Risk of Bias tool(14) a pre-specified analysis plan posted on Prospero, sensitivity analysis that address study quality and the assessment of publication bias.

\section{CONCLUSION}

In summary, data from six randomized controlled trials fail to demonstrate evidence of benefit from levothyroxine treatment for pregnancy outcomes in thyroid autoimmune women with normal thyroid function and subclinical hypothyroidism. In the absence of overt thyroid dysfunction, current trial evidence does not support the levothyroxine treatment of thyroid autoimmunity preconception or during pregnancy. Given the lack of evidence of benefit of levothyroxine therapy, it is time to re-examine recommendations for screening TPOAb and treatment of women with thyroid autoimmunity preconception or in pregnancy.

Acknowledgements. We thank Zahra Premji, PhD, MLIS (University of Calgary, Calgary, Alberta) for her expert advice on our search strategy.

Disclosure of interests: Authors have no conflicts of interest to disclose. 
Contribution to authorship: PL and LED conceived the idea for this systematic review and metaanalysis, and conducted the literature search along with the initial title/abstract review. JMY contributed her expertise in systematic review methodology to the design and conduct of this systematic review. LWL and JLB conducted the full text review, study selection, data extraction, and statistical analyses. LWL wrote the first draft of the manuscript. All authors contributed to critical review and approval of the final manuscript.

Ethics Statement. The authors adhered to the Preferred Reporting Items for Systemic Reviews and Meta-Analyses (PRISMA) guidelines. Ethical committee approval was not required for this study.

Funding: No funding sources were received or utilized for this study.

\section{REFERENCES}

1. Moreno-Reyes R, Glinoer D, Van Oyen H, Vandevijvere S. High prevalence of thyroid disorders in pregnant women in a mildly iodine-deficient country: a population-based study. J Clin Endocrinol Metab. 2013;98(9):3694-701.

2. McElduff AM, Jonathan. Thyroid function tests and thyroid autoantibodies in an unselected population of women undergoing first trimester screening for aneuploidy. The Australian \& New Zealand journal of obstetrics \& gynaecology. 2008;48(5):478-80.

3. Pratt DE, Kaberlein G, Dudkiewicz A, Karande V, Gleicher N. The association of antithyroid antibodies in euthyroid nonpregnant women with recurrent first trimester abortions in the next pregnancy. Fertil Steril. 1993;60(6):1001-5.

4. Kutteh WH, Yetman DL, Carr AC, Beck LA, Scott RT, Jr. Increased prevalence of antithyroid antibodies identified in women with recurrent pregnancy loss but not in women undergoing assisted reproduction. Fertil Steril. 1999;71(5):843-8.

5. Thangaratinam ST, Alex; Knox, Ellen; Kilby, Mark D.; Franklyn, Jayne; Coomarasamy, Arri. Association between thyroid autoantibodies and miscarriage and preterm birth: meta-analysis of evidence. BMJ (Clinical research ed). 2011;342:d2616.

6. Consortium on T, Pregnancy-Study Group on Preterm B, Korevaar TIM, Derakhshan A, Taylor PN, Meima M, et al. Association of Thyroid Function Test Abnormalities and Thyroid Autoimmunity With Preterm Birth: A Systematic Review and Meta-analysis. JAMA. 2019;322(7):632-41.

7. Stagnaro-Green A, Roman SH, Cobin RH, el-Harazy E, Alvarez-Marfany M, Davies TF. Detection of atrisk pregnancy by means of highly sensitive assays for thyroid autoantibodies. JAMA. 1990;264(11):1422-5.

8. Alexander EK, Pearce EN, Brent GA, Brown RS, Chen H, Dosiou C, et al. 2017 Guidelines of the American Thyroid Association for the Diagnosis and Management of Thyroid Disease During Pregnancy and the Postpartum. Thyroid. 2017;27(3):315-89.

9. Barton S. Which clinical studies provide the best evidence? The best RCT still trumps the best observational study. BMJ. 2000;321(7256):255-6.

10. GS HJ. Cochrane Handbook for Systematic Reviews of Interventions Version 5.1.0 [updated March 2011]. The Cochrance Collaboration. 2011.

11. Weeks I, Sturgess M, Siddle K, Jones MK, Woodhead JS. A high sensitivity immunochemiluminometric assay for human thyrotrophin. Clin Endocrinol (Oxf). 1984;20(4):489-95.

12. Nicoloff JT, Spencer CA. Clinical review 12: The use and misuse of the sensitive thyrotropin assays. J Clin Endocrinol Metab. 1990;71(3):553-8.

13. Cohen J. Weighted kappa: nominal scale agreement with provision for scaled disagreement or partial credit. Psychol Bull. 1968;70(4):213-20. 
14. Higgins JPT GS. Cochrane Handbook for Systematic Reviews of Interventions Version 5.1.0 [updated March 2011]. The Cochrane Collaboration, 2011. Available from http://handbook.cochrane.org.

15. Dhillon-Smith RKM, Lee J.; Sunner, Kirandeep K.; Cheed, Versha; Baker, Krys; Farrell-Carver, Samantha; Bender-Atik, Ruth; Agrawal, Rina; Bhatia, Kalsang; Edi-Osagie, Edmond; Ghobara, Tarek; Gupta, Pratima; Jurkovic, Davor; Khalaf, Yacoub; MacLean, Marjory; McCabe, Christopher; Mulbagal, Khashia; Nunes, Natalie; Overton, Caroline; Quenby, Siobhan; Rai, Raj; Raine-Fenning, Nick; Robinson, Lynne; Ross, Jackie; Sizer, Andrew; Small, Rachel; Tan, Alex; Underwood, Martyn; Kilby, Mark D.; Boelaert, Kristien; Daniels, Jane; Thangaratinam, Shakila; Chan, Shiao Y.; Coomarasamy, Arri. Levothyroxine in Women with Thyroid Peroxidase Antibodies before Conception. The New England journal of medicine. 2019;380(14):1316-25.

16. Nazarpour SRT, Fahimeh; Simbar, Masoumeh; Tohidi, Maryam; Alavi Majd, Hamid; Azizi, Fereidoun. Effects of levothyroxine treatment on pregnancy outcomes in pregnant women with autoimmune thyroid disease. European journal of endocrinology. 2017;176(2):253-65.

17. Negro RF, Gianni; Mangieri, Tiziana; Pezzarossa, Antonio; Dazzi, Davide; Hassan, Haslinda. Levothyroxine treatment in euthyroid pregnant women with autoimmune thyroid disease: effects on obstetrical complications. The Journal of clinical endocrinology and metabolism. 2006;91(7):2587-91.

18. Negro RM, Tiziana; Coppola, Lamberto; Presicce, Giovanni; Casavola, Eugenio Caroli; Gismondi, Riccardo; Locorotondo, Giancarlo; Caroli, Paolo; Pezzarossa, Antonio; Dazzi, Davide; Hassan, Haslinda. Levothyroxine treatment in thyroid peroxidase antibody-positive women undergoing assisted reproduction technologies: a prospective study. Human reproduction (Oxford, England). 2005;20(6):1529-33.

19. Negro RS, Alan; Stagnaro-Green, Alex. Impact of Levothyroxine in Miscarriage and Preterm Delivery Rates in First Trimester Thyroid Antibody-Positive Women With TSH Less Than $2.5 \mathrm{mIU} / \mathrm{L}$. The Journal of clinical endocrinology and metabolism. 2016;101(10):3685-90.

20. Wang HG, Hongwei; Chi, Hongbin; Zeng, Lin; Xiao, Wenhua; Wang, Yanrong; Li, Rong; Liu, Ping; Wang, Chen; Tian, Qing; Zhou, Zehong; Yang, Jin; Liu, Ye; Wei, Rui; Mol, Ben Willem J.; Hong, Tianpei; Qiao, Jie. Effect of Levothyroxine on Miscarriage Among Women With Normal Thyroid Function and Thyroid Autoimmunity Undergoing In Vitro Fertilization and Embryo Transfer: A Randomized Clinical Trial. JAMA. 2017;318(22):2190-8.

21. Casey BMT, Elizabeth A.; Peaceman, Alan M.; Varner, Michael W.; Sorokin, Yoram; Hirtz, Deborah G.; Reddy, Uma M.; Wapner, Ronald J.; Thorp, John M., Jr.; Saade, George; Tita, Alan T. N.; Rouse, Dwight J.; Sibai, Baha; Iams, Jay D.; Mercer, Brian M.; Tolosa, Jorge; Caritis, Steve N.; VanDorsten, J. Peter; Eunice Kennedy Shriver National Institute of Child, Health; Human Development Maternal-Fetal Medicine Units, Network. Treatment of Subclinical Hypothyroidism or Hypothyroxinemia in Pregnancy. The New England journal of medicine. 2017;376(9):815-25.

22. Lazarus JHB, Jonathan P.; Channon, Sue; Paradice, Ruth; Maina, Aldo; Rees, Rhian; Chiusano, Elisabetta; John, Rhys; Guaraldo, Varvara; George, Lynne M.; Perona, Marco; Dall'Amico, Daniela; Parkes, Arthur B.; Joomun, Mohammed; Wald, Nicholas J. Antenatal thyroid screening and childhood cognitive function. The New England journal of medicine. 2012;366(6):493-501.

23. Cappola AR, Casey BM. Thyroid Function Test Abnormalities During Pregnancy. JAMA. 2019;322(7):617-9.

24. Sun X, Hou N, Wang H, Ma L, Sun J, Liu Y. A Meta-Analysis of Pregnancy Outcomes with Levothyroxine Treatment in Euthyroid Women with Thyroid Autoimmunity. J Clin Endocrinol Metab. 2019.

25. Rao M, Zeng Z, Zhou F, Wang H, Liu J, Wang R, et al. Effect of levothyroxine supplementation on pregnancy loss and preterm birth in women with subclinical hypothyroidism and thyroid autoimmunity: a systematic review and meta-analysis. Hum Reprod Update. 2019;25(3):344-61. 
26. Rao M, Zeng Z, Zhao S, Tang L. Effect of levothyroxine supplementation on pregnancy outcomes in women with subclinical hypothyroidism and thyroid autoimmuneity undergoing in vitro fertilization/intracytoplasmic sperm injection: an updated meta-analysis of randomized controlled trials. Reprod Biol Endocrinol. 2018;16(1):92.

27. Abdel Rahman AH, Aly Abbassy H, Abbassy AA. Improved in vitro fertilization outcomes after treatment of subclinical hypothyroidism in infertile women. Endocr Pract. 2010;16(5):792-7.

28. Glinoer DR, M. [Thyroid changes in the pregnant woman]. Les alterations thyroidiennes chez la femme enceinte. 1993;54(6):385-8.

29. Vissenberg R, van Dijk MM, Fliers E, van der Post JAM, van Wely M, Bloemenkamp KWM, et al. Effect of levothyroxine on live birth rate in euthyroid women with recurrent miscarriage and TPO antibodies (T4LIFE study). Contemp Clin Trials. 2015;44:134-8.

Figure and Table Legend:

Figure 1. Preferred Reporting Items for Systematic Reviews and Meta-Analyses (PRISMA) Flow Diagram

Figure 2. Risk of bias assessment and trial quality assessment.

Figure 3. Results of meta-analysis of effects of levothyroxine treatment on pregnancy and obstetrical outcomes. (A) pregnancy achieved, (B) miscarriage (C) preterm delivery (D) live birth

Table 1. Characteristics of included randomized controlled trials. TSH, thyroid stimulating hormone; TPOAb, thyroid peroxidase antibody; LT4, levothyroxine; GA, gestational age; FT4, free thyroxine 


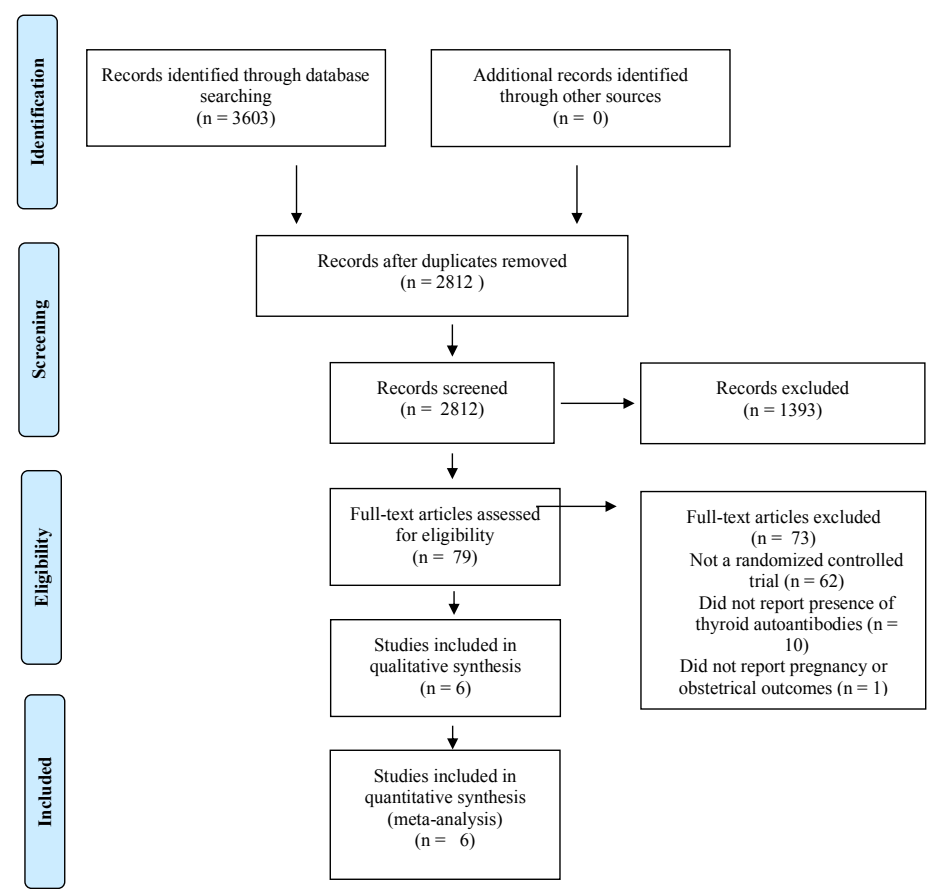

Figure 1. Preferred Reporting Items for Systematic Reviews and Meta-Analyses (PRISMA) Flow Diagram 

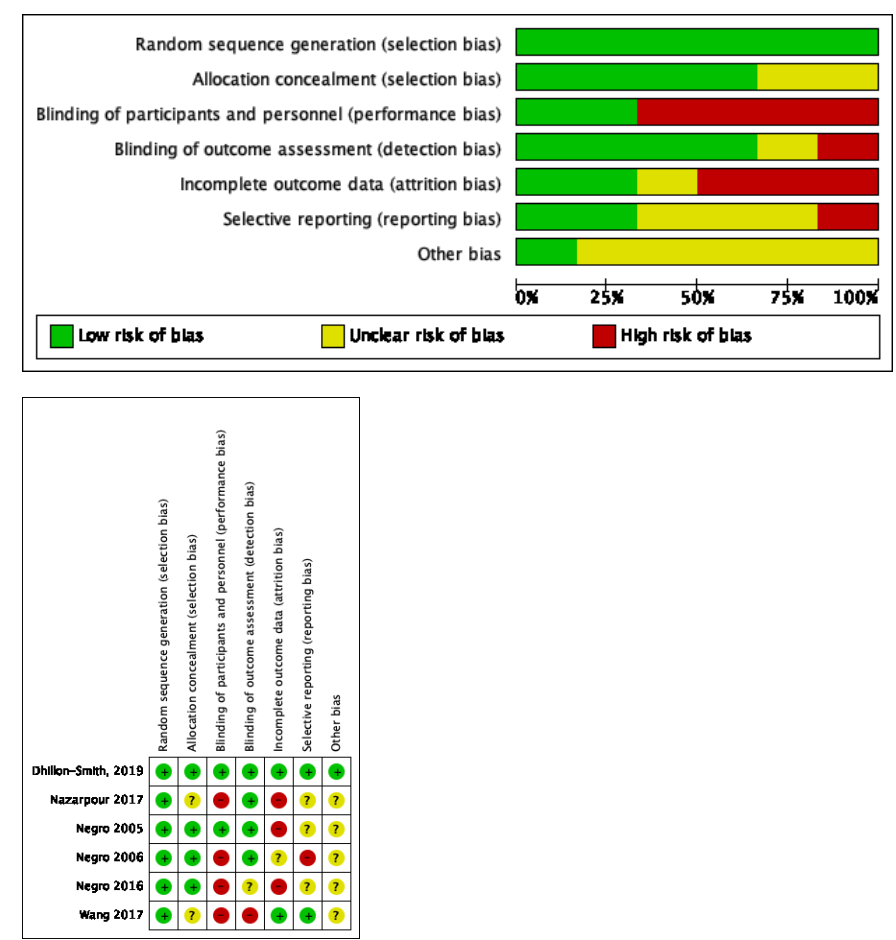

Figure 2. Risk of bias assessment and trial quality assessment. 

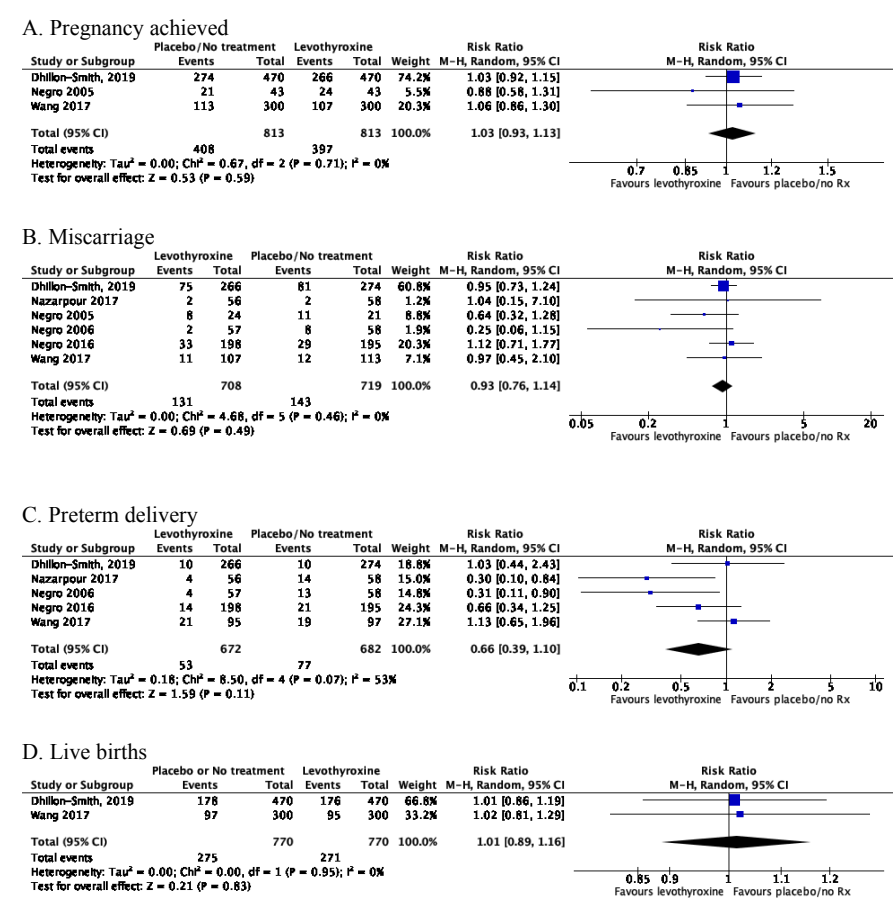

Figure 3. Meta-analysis of influence of levothyroxine on pregnancy outcomes. (A) pregnancy achieved, (B) miscarriage (C) preterm delivery (D) live birth

\section{Hosted file}

Main Table 1_July 142020 Final.docx available at https://authorea.com/users/345520/articles/ 471730-the-impact-of-levothyroxine-in-women-with-positive-thyroid-antibodies-onpregnancy-outcomes-a-systematic-review-and-meta-analysis-of-randomized-controlled-trials 